\title{
Periodic electroencephalographic patterns: usefulness of definition and classification
}

\author{
Padrões eletroencefalográficos periódicos: importância da definição e classificação
}

Eliana Garzon

MD; Coordinator of the

Electroencephalography Section of the Clinical Neurology Division of the Hospital das Clínicas of the São Paulo University Medical School.

\section{Correspondence}

Eliana Garzon

Avenida Doutor Enéas de Carvalho Aguiar 255 / PAMB / $6^{\circ}$ andar / Bloco 4

05403-900 São Paulo SP - Brasil E-mail:eliana.garzon@hcnet.usp.br

Conflict of interest

There is no conflict of interest to declare.

Received 04 December 2011 Accepted 12 December 2011
$\mathrm{F}$ ourteen years after Cobb and Hill ${ }^{1}$ first applied the term 'periodic' to characteristic electroencephalographic (EEG) changes, in 1950, Chatrian et al. ${ }^{2}$ identified a periodic and lateralized pattern, which they described as periodic lateralized epileptiform discharges (PLEDs). The acronym encapsulates the essential characteristics of PLEDs, namely, wide distribution, polymorphism, one or more sharp components in a repetitive way (usually at the rate of one to two seconds), and localization over the hemisphere. Subsequent studies have described other variable periodic patterns as two independent PLED types in the same EEG (BIPLEDs), multifocal PLEDs, generalized periodic epileptiform discharges (GPEDs), and stimulus-induced rhythmic, periodic, or ictal discharges (SIRPIDs) ${ }^{3-6}$.

PLEDs are usually observed in the context of acute, large, and destructive cortical processes, such as hemispheric stroke, supratentorial tumor, or encephalitis. Corresponding widespread lesions are thought to lead to a marginal zone of cortical hyperexcitability that serves as the 'pacemaker' for a spatially extended, synchronous EEG discharge ${ }^{7}$. Neuroimaging findings have demonstrated that while an acute cortical lesion is the most common structural substrate of PLEDs, subcortical lesions, chronic lesions, and nonlesional scans are not uncommon ${ }^{7}$. The spatially variable nature of brain lesions in PLEDs could point to their origin in variable segments of a cortical and subcortical 'system', assuming that it - whatever its precise components are - comprises a large-scale interconnected network, prone to synchronous oscillation springing from injury to any component?

Despite the frequent use of the term periodic, little attention has been paid to, or agreement reached on, how periodicity is defined or how the term should be used with respect to EEG phenomena. It has been suggested that the rate for a given patient should not vary more than $20 \%^{8}$. However, the method of measurement, the number of intervals that should be measured, and the putative clinical relevance of this value have not been discussed.

Most of these studies were retrospective, with EEGs being performed at arbitrary and widely varying time points in the course of the disease, with detailed information about these variables being provided in only a few instances. The importance of EEG timing in the detection of epileptiform abnormalities has not been generally appreciated, despite the fact that at least one study has clearly demonstrated that the incidence of periodic EEG patterns increases when they are performed earlier in the course of the disease process ${ }^{8,9}$.

Among the clinical manifestations, seizures are the most important and striking feature associated with PLEDs, occurring with a frequency from 58 to $100 \%^{2,8-11}$. This wide range probably reflects the heterogeneity of patient selection and study design. Focal motor seizures are usually seen in this clinical setting, often presenting as status epilepticus (epilepsia partialis continua) or as repetitive focal motor seizures ${ }^{2,8-11}$.

Some authors recognize PLEDs as being an ictal ${ }^{12,13}$ or peri-ictal ${ }^{14}$, phenomenon, due to their recurrent association with seizures. Electrographic criteria for defining a periodic pattern as an EEG seizure have also been proposed ${ }^{15}$.

Despite reports of regional increases in cerebral blood flow and oxygen use, and of hypermetabolism associated with PLEDs ${ }^{12,13}$, the clinical significance of periodic EEG patterns, whether PLEDs are an interictal or ictal activity, for instance, remains unclear.

In addition, there is still uncertainty surrounding how aggressively to treat with antiepileptic drugs (AEDs) patients exhibiting an EEG with a periodic pattern. The prognosis of patients with PLEDs is largely determined by the underlying disease process. Acute stroke appears to be associated with the worst prognosis, with mortality rates ranging from 28.8 to $53 \%^{16}$. Again, 
this wide variation probably reflects differences in patient selection between the studies, with the highest mortality rate seen in those with severe acute stroke. Potentially reversible conditions, such as metabolic encephalopathies and alcohol or barbiturate withdrawal, appear, by contrast, to have a much better prognosis ${ }^{16,17}$.

Against this background, the review article published by Andraus and colleagues ${ }^{18}$ in the current issue of Arquivos de Neuro-Psiquiatria provides evidence supporting the importance of recognizing periodic EEG patterns and their clinical significance. After an initial detailed discussion of the definition and EEG characterization of periodic patterns and their subtypes (PLEDs, BIPLEDs, GPEDs and SIRPIDs), an elaborate analysis of each subtype is separately made. The authors also discuss the nomenclature and present a glossary.

Regarding periodic patterns and their subtypes, one issue deserves attention. In 1991, a subclassification of PLEDs, based on the presence or absence of rhythmic discharges, was proposed ${ }^{18}$. The authors described brief and low amplitude focal stereotyped rhythmic discharges (RDs) closely associated (temporally and spatially) with higher amplitude interictal epileptiform discharges, and divided PLEDs into two categories: PLEDs plus, which were associated with RDs, and PLEDs proper, which were not. Correlation between seizures and RDs was higher when PLEDs-plus were observed.

This is an interesting study and we believe that the morphology of PLEDs is still an unsolved issue. Specific studies on morphologic variables, including duration of individual complexes, morphology stereotypy, degree of intervening slow rhythms, and periodicity need to be performed and compared across clinical settings.

Knowledge of the patterns and their subtypes is the key to right and precise interpretation, considering that periodic patterns are an epileptiform pattern with intriguing morphology, whose epileptogenic significance remains uncertain.

Periodic patterns are seen in a wide variety of etiologies, the discharges themselves being electrographically heterogeneous ${ }^{19}$. Consequently, patients should be carefully investigated for toxic-metabolic causes, infectious diseases, and/or intracranial lesions. Non-convulsive status epilepticus should also be considered. Recognition of these conditions is important to establish an accurate correlation of clinical, neurological, laboratory, and neuroimaging data with the EEG results. Analysis of observations should guide careful clinical decisions. A clinical trial with AEDs is possible, although experts should consider AED-related side effects and the special characteristics of the affected patients, who are generally in critical conditions and under intensive care. However, non-convulsive status epilepticus is one of several etiological possibilities, it is not the only one. It should also be considered that most patients will not respond clinically or electroencephalographically to AEDs.

This review article was valuable to show the importance and clinical significance of periodic EEG patterns, and to expose the strength of the data on which recommendations have been based. Since periodic patterns are considered controversial EEG patterns, not only this review useful is, but also future retrospective and prospective studies are highly desirable.

\section{References}

1. Cobb W, Hill D. Electroencephalogram in subacute progressive encephalitis. Brain 1950;73:392-404.

2. Chatrian GE, Shaw CM, Leffman H. The significance of periodic lateralized epileptiform discharges in EEG: an electrographic, clinical and pathological study. Electroencephalogr Clin Neurophysiol 1964;17:177-193.

3. de la Paz D, Brenner RP. Bilateral independent periodic lateralized epileptiform discharges. Clinical significance. Arch Neurol 1981;38:713-715.

4. Lawn ND, Westmoreland BF, Sharbrough FW. Multifocal periodic lateralized epileptiform discharges (PLEDs): EEG features and clinical correlations. Clin Neurophysiol 2000;111:2125-2129.

5. Brenner RP, Schaul N. Periodic EEG patterns: classification, clinical correlation, and pathophysiology.J Clin Neurophysiol 1990;7:249-267.

6. Hirsch LJ, Claassen J, MayerSA, Emerson RG.Stimulus induced rhythmic, periodic, or ictal discharges (SIRPIDs): a common EEG phenomenon in the critically ill. Epilepsia 2004;45:109-123.

7. Kalamangalam GP, Diehl B, Burgess RC. Neuroimaging and neurophysiology of periodic lateralized epileptiform eischarges: observations and hypotheses. Epilepsia 2007;48:1396-1405.

8. Schraeder PL, Singh N. Seizure disorders following periodic lateralized epileptiform discharges. Epilepsia 1980;21:647-654.

9. Snodgrass SM, Tsuburaya K, Ajmone-Marsan C. Clinical significance of periodic lateralized epileptiform discharges: relationship with status epilepticus.J Clin Neurophysiol 1989;6:159-172.

10. Kuroiwa Y,Celesia GG. Clinical significance of periodic EEG patterns. Arch Neurol 1980;37:15-20.
11. Young GB, Goodenough P, Jacono V, Schieven JR. Periodic lateralized epileptiform discharges (PLEDs): electrographic and clinical features. Am J EEG Technol 1988;28:1-13.

12. Handforth A, Cheng JT, Mandelkern MA, Treiman DM. Markedly increased mesiotemporal lobe metabolism in a case with PLEDs: further evidence that PLEDs are a manifestation of partial status epilepticus. Epilepsia 1994;35:876-881.

13. Ergün EL, Salanci BV, Erbas B, Saygi S. SPECT in periodic lateralized epileptiform discharges (PLEDs): a case report on PLEDs. Ann Nucl Med 2006;20:227-231.

14. Pohlmann-Eden B, Hoch DB, Cochius JL, Chiappa KH. Periodic lateralized epileptiform discharges: a critical review. J Clin Neurophysiol 1996;13:519-530.

15. Chong DJ, Hirsch LJ. Which EEG patterns warrant treatment in the critically ill? Reviewing the evidence for treatment of periodic epileptiform discharges and related patterns J Clin Neurophysiol 2005;22:79-91.

16. Walsh JM, Brenner RP. Periodic lateralized epileptiform discharges longterm outcome in adults. Epilepsia 1987;28:533-536.

17. Striano S, De Falco FA, Zaccaria F, Fels A, Narale S, Vacca G. Paroxysmal lateralized epileptiform discharges (PLEDs). Acta Neurol 1986;8:1-12.

18. Andraus MEC, Andraus CF, Alves-Leon S. Periodic EEG patterns: importance of their recognition and clinical significance Arq Neuropsiquiatr 2012;70:145-151.

19. Reiher J, Rivest J, Grand'Maison F, Leduc CP. Periodic lateralized epileptiform discharges with transitional rhythmic discharges: association with seizures. Electroencephalogr Clin Neurophysiol 1991;78:12-17. 